\title{
CORRELATIONS BETWEEN THE SEVERITY OF CARDIOVASCULAR AUTONOMIC NEUROPATHY AND OTHER COMPLICATIONS IN TYPE 2 DIABETES MELLITUS
}

\author{
Anca Motataianu', Zoltan Bajko, Smaranda Maier', Adina Stoian², \\ Laura Barcutean ${ }^{1}$, Rodica Balasa ${ }^{1}$ \\ ${ }^{1}$ Department of Neurology, University of Medicine and Pharmacy Targu-Mures, Romania \\ ${ }^{2}$ Department of Patophysiology, University of Medicine and Pharmacy Targu-Mures, Romania
}

\begin{abstract}
Introduction. Cardiovascular Autonomic Neuropathy (CAN), also known as the "silent killer", represents a serious complication of diabetes mellitus and is most of the times ignored. This is a highly topical issue, given the increased number of patients diagnosed with type 2 diabetes mellitus (T2DM).

The purpose of this study was to determine the association between the severity of CAN and the severity of other microvascular complications, such as diabetic sensory-motor polyneuropathy (DSMP), diabetic retinopathy (DR) and macrovascular complications (carotid and peripheral vascular atherosclerosis) in patients with T2DM.

Methods. A total of 149 patients with T2DM were assessed for CAN using the Ewing's battery of cardiovascular autonomic function tests. The patients were evaluated for diabetic microvascular complications (DSMP, RD), respectively macrovascular complications (carotid atherosclerosis by intima media thickness and peripheral vascular atherosclerosis by ankle-brachial index).

Results. Of the 45 patients with intermediate CAN, 35.55\% were without DR, $51.11 \%$ had non-proliferative DR and $13.33 \%$ had proliferative DR. From the 13 patients with severe CAN, $76.92 \%$ had non-proliferative DR and $23.07 \%$ had proliferative DR. From the 56 patients with subclinical CAN, $26.7 \%$ had no DSMP, $48.2 \%$ had subclinical DSMP and $25 \%$ had clinical DSMP. Of the 45 patients with intermediate CAN, $11.2 \%$ had subclinical DSMP and $80 \%$ had clinical DSMP.

Conclusion. In T2DM we demonstrated that an increase in the severity of cardiovascular autonomic injury is associated with an increase in the prevalence and severity of DSMP and DR, respectively carotid and peripheral atherosclerosis.
\end{abstract}

Keywords: cardiovascular autonomic neuropathy, type 2 diabetes mellitus, microvascular and macrovascular complications

\section{INTRODUCTION}

Diabetes mellitus (DM) is currently an extremely important health issue worldwide, given the high prevalence and the serious complications associated with DM, with a high mortality rate among these patients (1). Diabetic neuropathy (DN) is a frequent complication of T2DM, with an estimated prevalence of $8 \%$ in newly detected cases of diabetes and over $50 \%$ in cases with long evolution of the disease. Diabetic sensorimotor polyneuropathy (DSMP) with distal, symmetrical involvement and diabetic autonomic neuropathy (DAN) are the most common forms of DN $(2,3)$.

DAN is one of the least understood and recognized forms of DN, despite a significant negative impact upon the survival and quality of life of these patients. Given the importance of the autonomic 
nervous system in regulating the virtual function of each organ, the consequences of DAN may vary from troublesome or debilitating to catastrophic. Most of the internal organs receive dual innervation, sympathetic and parasympathetic. The vagus nerve is the longest autonomic nerve in the human body and mediates approximately $75 \%$ of all parasympathetic activity of the body. It is known that neuropathy occurs initially in the longest fibers, so the first manifestations of DAN will be parasympathetic. These manifestations are generalized, involving the gastrointestinal tract, urogenital tract and cardiovascular system $(4,5)$.

Cardiovascular autonomic neuropathy (CAN) represents the most important clinical form of DAN and is defined as impaired autonomic control of the cardiovascular system after excluding other causes. The autonomic nervous system closely integrates vital processes such as the heart rate, arterial blood pressure, myocardial contractility and therefore plays a key role in regulating the cardiovascular system. CAN is probably one of the most neglected serious complications of DM which occurs due to the affectation of autonomic fibers that innervate the heart and blood vessels, leading to cardiac rhythm and vascular dynamics impairment $(6,7,8,9)$.

CAN can cause orthostatic hypotension, physical exercise intolerance, high intraoperative risk due to the cardiocirculatory instability and last but not least, high incidence of silent myocardial infarctions, malignant cardiac arrhythmias and sudden deaths. The symptoms of CAN usually appear in the advanced stages of DM, but subclinical CAN can be detected in T2DM even in the first year after diagnosis, which highlights the importance of CAN screening $(4,10)$.

Several studies concluded that the presence of CAN substantially increases the risk of general mortality, cardiovascular mortality and major cardiovascular events. The CAN detection is important both for the clinical outcome and the prognosis of T2DM patients. Once CAN is identified, there is a need of adjusment of the physical exercise program of these patients, careful surveillance to identify silent myocardial ischemia, reassessment of chronic medication and aggressive treatment of associated cardiovascular risk factors $(11,12)$.

\section{MATERIAL AND METHOD}

This was a prospective study that included 149 patients diagnosed with T2DM, admitted to the Departments of Diabetology and Neurology of Targu
Mures Emergency County Hospital. We excluded patients with cardiac arrhythmias, symptomatic cardiac ischemic disease, heart failure and those with non-diabetic causes of autonomic and somatic neuropathy (multisystemic atrophy, Parkinson's disease, endocrine disorders, cancer, known B12 deficiency, porphyria, HIV infection, history of chemotherapy), patients with medication that affects the autonomic nervous system (anti arrhythmic drugs, tricyclic antidepressants, beta-blockers, diuretics such as spironolactone, antihistamine drugs, sympatomimetics), patients with poor glycemic control 24 hours before the autonomic tests, patients with acute vascular or non vascular diseases, patients with a chronic alcohol intake, morbid obesity (due to the impossibility to perform the parasympathetic tests), patients with chronic renal failure associated with uremic neuropathy and sympathetic hyperactivity, patients with chronic respiratory failure due to hypoxia and chronic hypercapnia which induce sympathetic activation.

All the patients included signed an informed consent form to participate in this study. The protocol for this study was approved by the Ethics Committee of the University of Medicine and Pharmacy of Targu Mures. The study was conducted in accordance with the Helinski Declaration guidelines.

\section{Clinical and electrophysiological evaluation of diabetic somatic sensory and motor neuropathy}

Following the standard guidelines, by interviewing each patient, we completed the symptoms questionnaire of diabetic neuropathy symptoms - Neuropathy Symptoms Score. The patients were objectively assessed by clinical neurological examination and by calculating the Neuropathy Disability Score, according to which they were classified as having mild, moderate or severe disability (13).

An electrophysiological study of motor and sensory nerve conduction was performed for each patient included in this study, in the Neuroelectrophysiology Laboratory of the Department of Neurology I from Targu-Mures, using a 4 channel NeuroMEP-Micro (Neurosoft) electromiography machine using standardized techniques with surface electrodes (14). All the tests were performed by the same examiner.

The motor nerve conduction study (NCS) was performed by bilateral supramaximal stimulation of the median, ulnar, peroneal and tibial nerves. Sensitive NCS was performed by bilateral antidromic stimulation of the median, ulnar and sural nerves. Nerve conduction was regarded modified when 
any of the conduction parameters (distal latency, amplitude or potential conduction velocity) were outside normal limits. When at least two nerves (peroneal nerve, tibial and sural) of the lower limbs, bilaterally, had abnormal electrophysiological values, NCS results were considered suggestive of diabetic neuropathy (15).

The patients were classified as follows:

- Absent DSMP: normal SCN

- Subclinical DSMP: abnormal SCN but in the absence of signs and symptoms for DSMP

- Clinical DSMP: abnormal SCN with signs and symptoms suggestive of clinical DSMP (16).

\section{Assessment of the cardiovascular autonomic neuropathy}

The cardiovascular autonomic tests were performed in most of the cases in the morning, in a quiet room with a stable and comfortable temperature $\left(22-24^{\circ} \mathrm{C}\right)$. The patients did not eat, did not smoke nor drink coffee at least 2 hours prior to the testing. The antidiabetic and chronic medication were administered at the end of the examination.

One examiner performed the cardiovascular autonomic tests for all of the patients, by using Ewing's method (17). The parasympathetic function was evaluated by analyzing the heart reat variability (HRV) to the Valsalva maneuver, to the deep breath test and to the supine to standing up test. $\mathrm{Pa}$ tients were monitored with a 12 derivation standard EKG and the HRV during the specified tests, was measured by automatic recording and determination of the R-R interval with the ELI 250 electrocardiograph computerized system (produced by Research Technology Inc./USA).

\section{A. Assessment of the HRV during the deep bre- athing test}

The patient is in supine position, connected to the computerized electrocardiograph which continuously records the heart rate. The patient breathes deeply for 5 seconds and exhales slowly for another 5 seconds, meaning a complete breath cycle at 10 seconds. The results of the test are expressed as a difference between the maximal and the minimal heart rate. A value between 11-14 beats/minute is considered a cutoff value, and $\leq 10$ beats/minute is considered abnormal $(17,18)$.

\section{B. Assessment of the HRV during the Valsalva maneuver}

The standard Valsalva maneuver was done with the patient in supine position, connected to the ELI 250 computerized electrocardiograph which continuously records the electrical heart activity. The patient is asked to perform a forced expiration with closed mouth and nose for 15 seconds, under a pressure of $40 \mathrm{mmHg}$. The quantitative value of the Valsalva maneuver, also known as the Valsalva coefficient, is calculated as the ratio between the longest R-R interval after the maneuver and the shortest R-R interval during the maneuver. There are no correlations between the values of the Valsalva coefficient and age and a value below 1.20 is considered abnormal $(17,19)$.

\section{Assessment of the HRV from supine to stan-}

\section{ding up test}

The patient is in supine position connected to the ELI 250 computerized electrocardiograph which continuously records the electrical heart activity and is asked to stand up without any assistance. After that we calculate the ratio 30:15, meaning the ratio between the longest R-R interval (approximmately at the 30th heartbeat) and the shortest R-R interval (approximmately at the 15th heartbeat). A value between 1.01-1.03 is a limit value and below 1.00 is abnormal (17).

\section{Assessment of the blood pressure variability} from supine to standing up test

The patient is in supine position for at least 5 minutes. The blood pressure is measured. After that, the patient is asked to stand up and the blood pressure is measured again after 2 minutes. If the systolic blood pressure drops between 11-29 $\mathrm{mmHg}$, it is considered a limit value, and if it drops more than $30 \mathrm{mmHg}$ is considered abnormal.

The global interpretation of the autonomic cardiovascular test results was the following:

Without $\mathrm{CAN}=$ all the tests were normal or one was at limit value;

Subclinical CAN $=$ one abnormal value in one of the $3 \mathrm{HRV}$ tests or 2 tests with limit values;

Intermediate $\mathrm{CAN}=$ two or more abnormal values in the HRV tests

Advanced CAN (severe impairment) $=$ two or more abnormal values in the HRV tests and abnormal blood pressure postural variability (20).

\section{Assessment of the intima-media thickness and the ankle-brachial index}

Each patient was evaluated by bilateral ultrasonography of the carotid arteries in order to determine the intima-media thickness (IMT). This examination was performed by the same specialist, using Siemens Accuson Antares Ultrasound System with a linear transducer VFX13-5 MHz after a standardized technique (21). All the patients included in the study were also evaluated by the ankle-brachial index (ABI) using a peripheral Doppler apparatus 
with a $5 \mathrm{mHz}$ Doppler probe (Hi Dop Vascular Doppler set).

\section{Statistical analysis}

The statistical analysis of the data was performed by using the MedCalc program for statistics in biomedical research. The descriptive data and variable distribution were described by using summary statistics or frequency analysis. We used $\chi^{2}, \mathrm{t}-\mathrm{Stu}-$ dent, Anova and Pearson correlation tests.

\section{RESULTS}

\section{Correlation between CAN and DSMP}

Of the 149 patients, 35 patients had no CAN, 56 had subclinical CAN, 45 had intermdiate CAN and 13 had severe CAN. Of the 35 patients with no CAN, 24 had no DSMP (68.5\%) and 11 had subclinical DSMP (31.5\%). Of the 56 patients with subclinical CAN, 15 had no DSMP (26.7\%), 27 had subclinical DSMP (48.2\%) and 14 (25\%) had clinical DSMP. Of the 45 patients with intermediate CAN, 4 (8.8\%) had no DSMP, 5 (11.2\%) had subclinical DSMP and $36(80 \%)$ had clinical DSMP. All 13 patients with severe CAN had clinical DSMP. We used $\chi^{2}$ test between 2 quantitative variables and found a statistical significance with the $p$ value - 0,0001 .

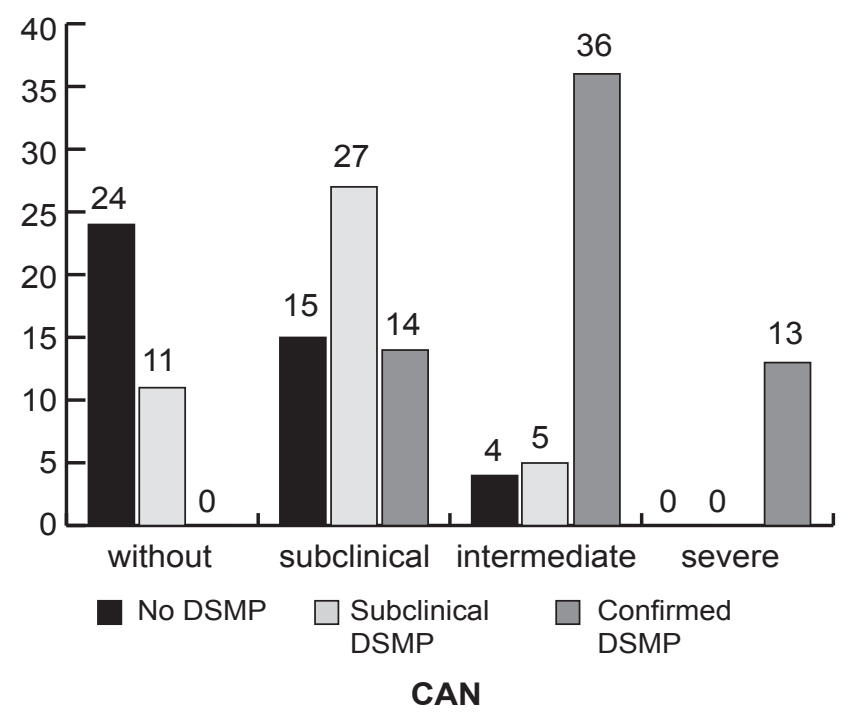

FIGURE 1. DSMP prevalence according the CAN severity in T2DM patients $(p=0.0001)$

\section{Correlation between CAN and diabetic retinopathy}

The patients with no CAN (13) had no diabetic retinopathy (DR). Of the 56 patients with subclinical CAN, $45(80.35 \%)$ were free of DR, $10(17.85 \%)$ had non proliferative DR and 1 patient (1.78\%) had proliferative DR. Of the 45 patients with intermediate CAN, $16(35.55 \%)$ were free of DR, $23(51.11 \%)$ had non proliferative DR and $6(13.33 \%)$ had proliferative DR. Of the 13 patients with severe CAN, $10(76.92 \%)$ had non proliferative DR and 3 (23.07\%) had proliferative DR.

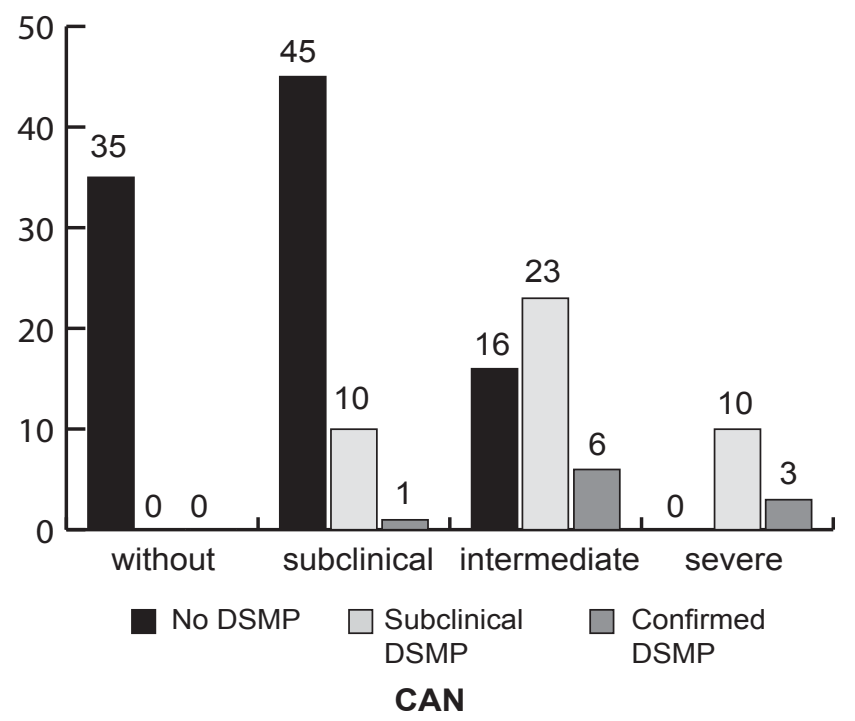

FIGURE 2. Diabetic rethinopathy prevalence according to CAN severity in T2DM patients $(p=0.0001)$

\section{Correlation between $\mathrm{CAN}$ and $\mathrm{ABI}$}

After statistical analysis using the ANOVA test between the ABI average values of the 4 types of CAN we noticed that the average values of ABI dropped significantly from the group of patients with no CAN to the ones with severe CAN (p-0.0001). Therefore, in patients with no CAN, the average value of ABI was $1.047 \pm 0.04$, in the patients with subclinical CAN it was $0.9548 \pm 0.08$ and in patients with intermediate CAN it was $0.9207 \pm 0.166$. In the group of patients with severe/ advanced CAN, the average value of $\mathrm{ABI}$ was $0.8700 \pm 0.2068$.

By using the multiple comparison Bonferroni test, we see that there is a significant statistical difference between the group of patients with no CAN compared to the ones with subclinical CAN $(p<0.05)$, between the group with no CAN and confirmed CAN $(p<0.05)$ and between the group with no CAN and severe CAN $(\mathrm{p}<0.05)$.

\section{Correlation between CAN and IMT}

By using the ANOVA test between the average values of the IMT corresponding to the 4 types of CAN, we noticed that the average values of IMT significantly increase from the group without CAN to the one with severe CAN (p-0.0001). Therefore, 


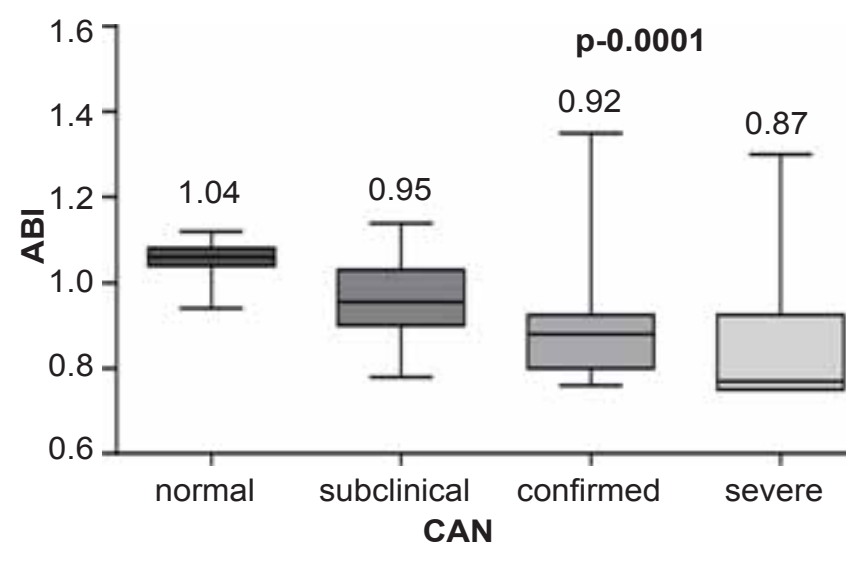

FIGURE 3. The ABI in T2DM patients according to CAN severity

in patients with no CAN the average IMT is $0.7100 \pm 0.13$, in patients with subclinical CAN is $0.9046 \pm 0.1567$, in patients with intermediate CAN is $1.006 \pm 0.138$ and in patients with advanced CAN is $1.155 \pm 0.1650$.

By using the multiple comparisson Bonferroni test, we see that there is a significant statistical difference between the group with no CAN vs subclinical CAN $(p<0.05)$, between the group with no CAN vs confirmed CAN $(p<0.05)$, between the group without CAN vs severe CAN $(\mathrm{p}<0.05)$, between the group of subclinical CAN vs intermediate CAN, between the group of subclinical CAN vs severe CAN and between the group of intermediate CAN vs severe CAN.

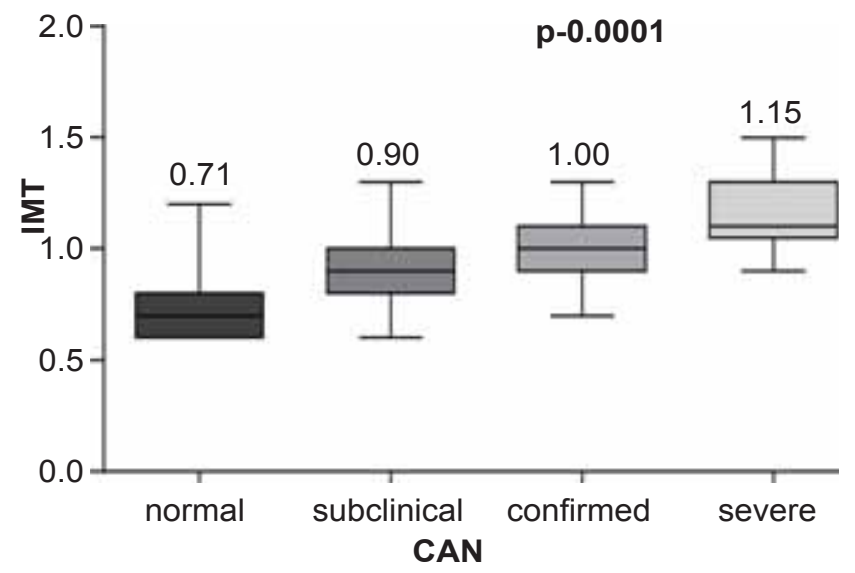

FIGURE 4. IMT values in T2DM patients according to CAN severity

\section{DISCUSSIONS}

In our study, we noticed that there is a significant association between the presence of somatic neuropathy confirmed by using electrophysiological study and the severity of CAN. Therefore, we observed that an increase in severity of cardiovas- cular autonomic injury is accompanied by increased severity of sensory and motor fiber damage in T2DM. This association can be explained by a greater accuracy and sensitivity in detecting DSMP by using electrophysiological studies. This data is in accordance with the studies performed by Bilal et al (22).

The results of our study might be considered somehow surprising, due to the fact that the presence of CAN implies the damage of the thin, unmyelinated fibers that are responsible for the autonomic cardiovascular control, while the DSMP, as evaluated by electrophysiological studies, implies the damage of the well myelinated, thick sensory and motor nerve fibers. This association between the severity of CAN and DSMP as assessed by electrophysiological studies was also reported by other authors. We can affirm that these 2 complications evolve at the same time, that there is no difference in hyperglicemia induced vulnerability of the two types of nerve fibers and that they can be damaged at the same time $(23,24)$.

In a study conducted by Valensi et al (25) it was observed that there was an independent association between DSMP (as assessed by electrophysiological studies) and retinopathy, also between DSMP and diabetic nephropathy. In the same study it was noted that the duration of DM was a major contributor in developing these complications compared to glycemic control, which suggested that vascular factors play an important role along with the metabolic factors in the development of diabetic neuropathy.

A high incidence of CAN was reported in patients with proliferative DR or diabetic nephropathy. In a study conducted by Valensi et al (26) it was observed that there is a statistical significant association between DR as assessed by angiofluorography and CAN. In their study, more than half of the patients without DR had CAN. These results are consistent with those obtained in our study and they support the hypothesis that parasympathetic autonomic cardiovascular dysfunction usually precedes the onset of DR.

By detecting CAN in more than half of the patients without DR and finding severe disorders (proliferative DR) in patients with both sympathetic and parasympathetic impairment (advanced CAN) it suggests that autonomic cardiovascular function damage appears earlier and can be a contributing factor for progression of microvascular complications in T2DM patients $(27,28)$.

In another study it was demonstrated that vagal dysfunction was associated with diabetic nephro- 
pathy. Our study data reveals that the severity of autonomic cardiovascular damage is associated with an increased prevalence of DSMP and DR in T2DM. These findings suggest that parasympathetic autonomic dysfunction can induce blood flow changes in the microcirculation. The reduction of sympathetic vasoconstrictor tonus can increase microcirculatory blood flow, inducing and exacerbating capillary permeability function, thus facilitating the deposition of glycosilated proteins on the basal membrane and capillary damage. The association between the microvascular complications suggests the existence of a common pathogenic mechanism. It has been demonstrated that hyperglycemia is the key factor which determines an increase of the oxidative stress, subsequently leading to endothelial dysfunction $(28,29,30)$.

Diabetic patients have an atherosclerotic process defined by an early onset and an accelerated evolution. DM increases the risk of peripheral arterial disease by 2-3 times. Peripheral arterial disease, together with DSMP are responsible for the high incidence of non-traumatic limb amputation in diabetic patients. $\mathrm{ABI}$ is a specific and sensitive test used to determine the atherosclerotic process in the periphery. In our study, we noticed that as the autonomic cardiovascular damage increases, the average value of ABI decreases. Also, as the autonomic cardiovascular damage increases, the IMT increases. These results confirm the existing connection between the CAN and incipient atherosclerosis. Chronic inflammation and endothelial dysfunction represent an early stage of the atherosclerotic process $(31,32)$.

Carotid atherosclerosis is often accompanied by coronary and peripheral artery atherosclerosis (33-35).

In a study published by Gottsater et al (36), it was demonstrated that T2DM patients with CAN have carotid atherosclerosis more frequently than those with no CAN and that both CAN and carotid atherosclerosis were progressive 8 years after the diagnosis of T2DM despite an adequate control of the metabolic parameters (HbA1c, IMT, lipid profile).

In the ARIC study on patients with T2DM, the investigators tried to explain the link between CAN and the accelerated atherosclerotic process. It was discovered that patients with a decrease in the HRV often had a more accentuated coronary artery atherosclerosis, which was not dependent on the presence of conventional risk factors. In patients with T2DM, autonomic neuropathy is involved in the acceleration of the atherosclerotic process. Arterial remodeling and stiffness represent the connection between cardiovascular risk factors and atherogenesis. Vascular remodelation, together with an increase of the IMT index, followed by arterial distension, the so called early arterial stiffness, can be detected early in diabetic patients $(37,38)$.

In our study, the relationship between the severity of DCAN and IMT index increase and an ABI decrease demonstrates that the autonomic cardiovascular damage is also associated with the development of macrovascular complications.

Endothelial dysfunction plays a key role in the occurrence of micro and macrovascular complications. Endothelial dysfunction is considered to be a forerunner of atherosclerosis. When vasodilator dysfunction induced by endothelial cell is present, a proinflammatory and procoagulant status is present which will determine the genesis of the atherosclerotic plaques. When the endothelial function is restored, it is possible that an anti-atherosclerotic process will reduce the cardiovascular complications. An increase in the oxidative stress, which appears secondary to the hyperglycemia plays a decisive role in the activation of the multifactorial mechanisms that will set in motion the endothelial dysfunction (39).

Our study demonstrated the association between the severity of CAN and microvascular complications in T2DM, also between CAN and macrovascular complications. Both these complications have a common pathogenic mechanism. In the center of this unifying theory of complication development lays the endothelial dysfunction, which explains both the genesis of each complication and their further progression. This seems to be the common pathogenic mechanism that links together the vascular complications, apparently „distant” of T2DM.

\section{CONCLUSIONS}

There is an important association between the presence of confirmed DSMP (by electrophysiological studies) and the severity of CAN. Increased severity of the cardiovascular autonomic system injury is accompanied by increased severity of sensory-motor fiber damage in T2DM. We can say that there is no difference in vulnerability between the two types of nervous fibers (autonomic and somatic) to hyperglycemic action, thus these two types of fibers can be damaged at the same time in DM. In T2DM we demonstrated that an increase in severity of cardiovascular autonomous injury is associated with an increase in prevalence and severity of 
DSMP and DR, also carotid and peripheral atherosclerosis.

Parasympathetic autonomic cardiovascular dysfunction usually precedes clinical diagnosis of the other microvascular complications of diabetes, especially diabetic retinopathy, and this autonomic cardiovascular injury might be a contributing factor for the appearance and progression of the other microvascular complications. CAN can thus be considered a prognostic indicator in T2DM evolution and a predictor of the presence and severity of other micro and macrovascular complications. These re- sults underline the necessity of complete screening for complications associated with DM, especially in patients with CAN, where the diagnosis of a diabetic complication implies the necessity of a full work-up in order to detect the other micro and macrovascular complications.

\section{Acknowledgement}

This study was supported by the internal research grant of the University of Medicine and Pharmacy Targu-Mures, grant number 18/2015.

\section{REFERENCES}

1. Rett K. Diabetes Therapy on the Move: Is glucocentric view outdated? In: Rett K (Ed). Better chances for nerves and vessels, Georg Thieme Verlag KG Stuttgart 2011:1-8.

2. Boulton A.J., Vinik A.I., Arezzo J.C. et al. Diabetic neuropathies: a statement by the American Diabetes Association. Diabetes Care 2005; 28: 956-962.

3. Shakher J., Stevens M.J. Update on management of diabetic polyneuropathies. Diabetes Metab Syndr Obes 2011; 4: 289305.

4. Edwards J.L., Vicent A.M., Cheng H.T. et al. Diabetic neuropathy: mechanism to management. Pharmacol Ther 2008; 120: 1-34.

5. Vinik A.I., Erbas T. Diabetic autonomic neuropathy. In: Bujs R, Swaab D (Eds). Handbook of Clinical Neurology. Autonomic Nervous System, Elsevier, Amsterdam 2013; 117: 279-294.

6. Kempler P. Cardiac autonomic neuropathy: Is it a cardiovascular risk factor in type 2 diabetes? In: Hancu N. (Ed). Cardiovascular Risk in Type 2 Diabetes Mellitus: assessment and control, Springer-Verlag, Berlin Heidelberg 2003:181-192.

7. DCCT Research Group. The effect of intensive diabetes therapy on measures of autonomic nervous system function in the Diabetes Control and Complications Trial (DCCT). Diabetologia 1998; 41: 416-423.

8. Pop-Busui R. What do we know and we do not know about cardiovascular autonomic neuropathy. J Cardiovasc Transl Res 2012; 5: 463-478.

9. Vinik A.I., Maser R.E., Mitchell B.D. et al. Diabetic autonomic neuropathy. Diabetes Care 2003; 26: 1553-1579.

10. Vinik A.I., Ziegler D. Diabetic cardiovascular autonomic neuropathy. Circulation 2007; 115: 387-397.

11. Maser R.E., Lenhard M.J. Cardiovascular autonomic neuropathy due to diabetes mellitus: clinical manifestations, consequences and treatment. J Clin Endocrinol Metab 2005; 90: 5896-5903.

12. Freeman R. Autonomic peripheral neuropathy. Lancet $2005 ; 365$ : 1259-1270.

13. Young M.J., Boulton A.J., MacLeod A.F. et al. A multicentre study of the prevalence of diabetic peripheral neuropathy in the United Kingdom hospital clinic population. Diabetologia 1993; 36: 150-154.

14. Lew H.L., Tsai S.J. Technical Aspects of EMG - pictorial guide to nerve conduction techniques. In: Pease W.S., Lew H.L., Johnson E.W. (Eds). Practical Electromyography. Lippincott Williams \& Wilkins, Philadelphia, Pennsylvania 2007: 213-255.

15. Dyck P.J. Detection, characterization and staging of polyneuropathy: assessed in diabetics. Muscle and Nerve 1988; 11: 21-32.

16. Tesfaye S., Boulton A.J., Dyck P.J. et al. Diabetic Neuropathies: update on definitions, diagnostic criteria, estimation of severity, and treatments. Diabetes Care 2010; 33: 2285-2293.

17. Ewing D.J., Martyn C.N., Young R.J. et al. The value of cardiovascular autonomic function tests: 10 years experience in diabetes. Diabetes Care 1985; 8:491-498.

18. Pfeifer M.A., Cook D., Brodsky J. et al. Quantitative evaluation of cardiac parasympathetic activity in normal and diabetic man. Diabetes 1982; 31:339-345.

19. Ewing D.J. Autonomic neuropathy. In: Pickup C, Williams G (Eds). Chronic complications of diabetes, Blackwell Scientific Publications, Oxford 1994: 124-136.

20. Vinik A.I., Maser R.E., Mitchell B.D. et al. Diabetic autonomic neuropathy. Diabetes Care 2003; 26: 1553-1579.

21. Wendelhag I., Gustavsson T., Suurkula M. et al. Ultrasound measurement of wall thickness in the carotid artery: fundamental principles and description of computerized image analyzing system. Clin Physiol 1991; 11: 565-577.

22. Bilal N., Erdogan M., Ozbek M. et al. Increasing severity of cardiac autonomic neuropathy is associated with increasing prevalence of nephropathy, retinopathy and peripheral neuropathy in Turkish type 2 diabetics. J Diabetes Complications 2008; 22: 181-185.

23. Pfeifer M.A., Weinberg C.R., Cook D.L. et al. Correlations among autonomic, sensory and motor neural function tests in untreated non-insulin dependent diabetic individuals. Diabetes Care 1985; 8: 576-584.

24. Young R.J., Zhou Y.Q., Rodriques E. et al. Variable relationship between peripheral somatic and autonomic neuropathy in patients with different syndromes of diabetic polyneuropathy. Diabetes 1986; 35: 192-197.

25. Valensi P., Giroux C., Seeboth-Ghalayini B. et al. Diabetic peripheral neuropathy: effects of age, duration of diabetes, glycemic control and vascular factors. J Diabetes Complications 1997; 11: 27-34.

26. Valensi P., Paries J., Attali J.R. et al. Cardiac autonomic neuropathy in diabetic patients: influence of diabetes duration, obesity and microangiopathic complications- the French multicenter study. Metabolism 2003; 52: 815-820.

27. Dyrberg T., Benn J., Christiansen J.S. et al. Prevalence of diabetic autonomic neuropathy measured by simple bedside tests. Diabetologia 1981; 20: 190-194.

28. Brahimi M., Dabire H., Platon P. et al. Arterial rigidity and cardiovascular vagosympathetic activity in normotensive and hypertensive obese patients and type 2 diabetics. Arch Mal Coeur Vaiss 2001; 94: 944-946.

29. Brownlee M. Biochemistry and molecular cell biology of diabetic complications. Nature 2000; 414: 813-820. 
30. Valensi P., Attali J.R., Behar A. et al. Increased capillary permeability to albumin and diabetic neuropathy. Diabetes Res Clin Pract 1991; 11: 41-46.

31. Diamant M., Lamb H.J., Smit J.W. et al. Diabetic cardiomyopathy in uncomplicated type 2 diabetes is associated with the metabolic syndrome and systemic inflammation. Diabetologia 2005; 48: 1669-1670.

32. Andel M., Polak J., Kraml P. et al. Chronic mild inflammation links obesity, metabolic syndrome, atherosclerosis and diabetes. Vnitr Lek 2009; 55: 659-665.

33. Mack W.J., LaBree L., Liu C. et al. Correlations between measures of atherosclerosis change using carotid ultrasonography and coronary angiography. Atherosclerosis 2000; 150: 371-379.

34. Cheng S.W., Wu L.L., Ting A.C. et al. Screening for asymptomatic carotid stenosis in patients with peripheral vascular disease: a prospective study and risk factor analysis. Cardiovascular Surg 1999; 7: 303-309.

35. Simons P.C., Algra A., Eikelboom B.C. Carotid artery stenosis in patients with peripheral arterial disease: the SMART study. J Vasc Surg 1999; 30: 519-525.
36. Gottsater A., Szelag B., Berglund G. et al. Changing associations between progressive cardiovascular autonomic neuropathy and carotid atherosclerosis with increasing duration of type 2 diabetes mellitus. J Diabetes Complications 2005; 19: 212-217.

37. Liao D., Carnethon M., Evans G.W. et al. Lower heart rate variability is associated with the development of coronary heart disease in individuals with diabetes:the atherosclerosis risk in communities (ARIC) Study. Diabetes 2002; 51: 3524-3531.

38. Rodriques T.C., Ehrlich J., Hunter C.M. et al. Reduced heart rate variability predicts progression of coronary artery calcification in adults with type 1 diabetes and controls without diabetes. Diabetes Technol Ther 2010; 12: 963-969.

39. Nystrőm T., Nygren A., Sjoholm A. Persistent endothelial dysfunction is related to elevated $C$ reactive protein (CRP) levels in type II diabetic patients after acute myocardial infarction. Clin Sci 2005; 108: 121-128. 